\title{
Correction to: Sentiment Analysis of Stock Market Investors and Its Correlation with Stock Price Using Maximum Entropy
}

Liang Xue, Han Wang, Fengling Wang, and Huawei Ma

\section{Correction to:}

Chapter "Sentiment Analysis of Stock Market Investors and Its Correlation with Stock Price Using Maximum Entropy" in: R. Lee (ed.),

Computer and Information Science 2021-Summer, Studies in Computational Intelligence 985, https://doi.org/10.1007/978-3-030-79474-3_3

The original version of the book was published with incorrect spelling for corresponding author name as "Huawen Ma" and now this has been updated as "Huawei Ma" in the chapter "Sentiment Analysis of Stock Market Investors and Its Correlation with Stock Price Using Maximum Entropy”. The chapter and book have been updated with the changes. 